Case Report

\title{
Impact of Osteophytectomy on Swallowing Function in a Patient with Chronic Dysphagia
}

Melissa M Howard, Justin Phillips, Stefan Henley, Sarah E. Green, Emily R. Rosario *

Acute Rehabilitation, Casa Colina Hospital and Centers for Healthcare Pomona, California, USA; EMails: mhoward@casacolina.org; jphillips@casacolina.org; shenley@casacolina.org; sgreen@casacolina.org; erosario@casacolina.org

* Correspondence: Emily Rosario; E-Mail: erosario@casacolina.org

Academic Editor: David G Smithard

Collection: Dysphagia in the Elderly

OBM Geriatrics

2021, volume 5, issue 3

doi:10.21926/obm.geriatr.2103175
Received: November 01, 2020

Accepted: April 07, 2021

Published: July 07, 2021

\begin{abstract}
Anterior cervical osteophytes are common in the geriatric population. Dysphagia can occur in individuals suffering from these spinal abnormalities. Surgical intervention is an uncommon course of treatment for these patients, but is often utilized as a last resort with the hope of swallow recovery. The purpose of this article is to highlight a unique case study documenting the required treatment course for dysphagia associated with osteophytes and subsequent osteophytectomy. We review current literature of both conservative and surgical interventions, as well as discuss the rehabilitation course for a complex patient with persistent dysphagia. Various outcome measures were utilized during the patient's inpatient stay to track progress including the Functional Oral Intake Scale (FOIS), the Bolus Residue Scale (BRS), Penetration Aspiration Scale (PenAsp), Dysphagia Outcome Severity Scale (DOSS), and a Modified Barium Swallow Study (MBSS). The patient received rehabilitative training including oral motor and pharyngeal strengthening exercises, respiratory strengthening, speech instruction, cognitive retraining, and instrumental assessment. Following osteophytectomy and dysphagia rehabilitation the patient did not show any change based on a repeat MBSS which revealed the necessity for the patient to remain nothing per oral (NPO). The patient
\end{abstract}

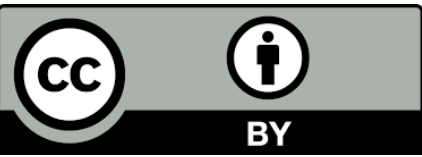

(C) 2021 by the author. This is an open access article distributed under the conditions of the Creative Commons by Attribution License, which permits unrestricted use, distribution, and reproduction in any medium or format, provided the original work is correctly cited. 
demonstrated an inability to manage his secretions, requiring continual suctioning. Upon discharge the patient remained NPO with the exception of ice chips, utilized a PEG for nutrition, and had a red capped tracheostomy. He was on room air and independently utilized oral suction as needed for secretion management. Our patient's clinical course was not aligned with typical osteophytectomy recovery as progress after his 25-day inpatient stay was limited. The goal of this case study is to contribute information to the limited and variable data available regarding treatment options, outcome measures and timelines for recovery as it pertains to patients who undergo an osteophytectomy.

\section{Keywords}

Dysphagia; swallow; osteophyte; osteophytectomy; spine; surgery

\section{Introduction and Literature Review}

Anterior cervical osteophytes (ACOs) are bony protrusions of the spine seen primarily in the geriatric male population, often those 65 years and older, as a result of degenerative spinal changes, ankylosing spondylitis, or diffuse idiopathic skeletal hyperostosis (DISH) [1-6]. It is estimated that ACOs occur in 10-30\% of the population, are typically benign, and are most often asymptomatic [7, 8]. According to Egerter et al. [7], age seems to correlate with the severity of symptoms. In some individuals ACOs compress the pharynx and/or esophagus or cause soft tissue inflammation narrowing the nasopharynx resulting in dysphonia, dyspnea, and/or dysphagia [4, 6, 9]. Although additional research is needed to determine the exact mechanism impacting the presence of ACOs, Choi et al. [2] suggests that C3-5 is most often where dysphagia secondary to ACOs occurs. Physiologically, this is where epiglottic inversion for airway protection occurs which is highly correlated with the process of swallowing.

For individuals affected by ACOs, dysphagia typically begins with solids and eventually progresses to liquid consistencies. Individuals may initially report sticking of food in their throat that eventually leads to the need for softer solids and frequent liquid intake to help clear pharyngeal residue. If the dysphagia is not addressed patients begin to notice prolonged meals and subsequent weight loss, resulting in decreased quality of life $[1,5,7]$. Additionally if severe the dysphagia places patients at risk for aspiration. The mainstay of treatment for dysphagia caused by cervical osteophytes is conservative with diet modification, swallowing strategies, and anti-inflammatories [10].

In an interdisciplinary team approach to ACOs and dysphagia, a medical speech language pathologist (SLP) is responsible for the assessment of oropharyngeal swallowing, recommendations for diet modification, and prescription of dysphagia therapy [11]. Current literature suggests the importance of extensive diagnostic measures by a multidisciplinary team, including the completion of a MBSS to confirm presence of ACOs and exclude other causes for dysphagia [6, 12-16]. An MBSS is a radiographic instrumental assessment of the structures and functions of the oral and pharyngeal cavities. It assesses the effects of intubation, mechanical ventilation, neurologic and/or respiratory compromise, and surgical interventions on swallow function. It also looks at swallow safety based on airway protection, bolus size and consistency, the impact of therapeutic techniques on bolus management as well as screening esophageal motility [11]. 
There is limited evidence to support specific interventions implemented by SLPs pre- and postsurgical intervention. The current literature consists primarily of case reports, case series, and retrospective cohort studies with limited participants. One case study conducted by Jeong et al. [17] reported daily dysphagia therapy including oral and pharyngeal strengthening such as the Shaker exercise and therapeutic maneuvers such as the chin tuck and supraglottic swallow. Carlson et al. [18] also reported the chin tuck to be successful for patients experiencing dysphagia secondary to ACOs. Other conservative intervention approaches include the use of anti-inflammatory drugs and muscle relaxants, which can offer temporary relief of dysphagia symptoms [7-9, 19, 20].

A small percentage of patients require surgical intervention when conservative measures fail and worsening of dysphagia or dyspnea symptoms occur. Often, surgical intervention is not pursued for those in the geriatric population due to risks related to patients' prior comorbidities or post-surgical concerns regarding soft tissue edema and laryngeal nerve damage [2]. Osteophytectomy is performed under general anesthesia and consists of anterolateral and posterolateral approaches, both of which have been found to have certain advantages. Occasionally, a discectomy or spinal fusion may also be necessary [5]. For some patients, recurrence of ACOs is possible. Post-surgical complications have been reported in approximately $10 \%$ of individuals, with prolonged tracheostomy being an additional complication that is less common [6]. One study found that all patients requiring tracheostomy were decannulated by 2 months post-surgery [5].

Additional studies have shown large variability in recovery of swallow function postoperatively, ranging from one day after surgery to marked improvement noted at six months $[5,7]$. There has also been variability in terms of individuals resuming oral intake. While Lecerf et al. [5] found that one individual resumed intake the same day of surgery, Mallepally et al. [14] found intake to resume one day post-surgery. Lecerf et al. also found normal eating to resume for one individual one-month post-surgery, whereas Carlson et al. [18] observed that dysphagia symptoms required three months or more to subside. Egerter et al. [7] noted significant improvement six months after surgery was completed. This range of variability could be related to age, comorbidities, location of osteophyte, thickness of osteophyte, complexity of the surgery, or postoperative edema[21, 22], which in turn makes prognostication difficult.

In this case report the authors review a patient's course with chronic dysphagia as a result of ACOs and persistent dysphagia during his 25-day inpatient rehabilitation stay, status post an osteophytectomy. In this unique case, this individual with an unusual medical history had an osteophytectomy after a 4-year battle with dysphagia. His goal was to ease his per oral (PO) intake and improve his quality of life. This case report will review swallowing issues associated with osteophytes, discuss current literature regarding osteophytes and osteophytectomy, and discuss other treatment options and rehabilitation after an osteophytectomy. The goal is to increase knowledge of this surgical intervention and the complexities surrounding the rehabilitation course, not only for the speech language pathology community, but also the geriatric population suffering from this anatomical abnormality. The authors anticipate that insight will be gained regarding progress or lack of progress in the early stages after surgery.

\section{Clinical Case}

The patient is a 73-year-old male with prior history of severe sleep apnea, obesity, and uvulopalatopharyngoplasty 15 years prior, who had been experiencing progressively worsening 
dysphagia for approximately 4 years. He was also noted to have a history of right carotid endarterectomy 14 years prior, but no history of TIA or stroke. He was seen by neurosurgery in clinic and on neurologic exam had no focal neurologic deficits aside from his dysphagia. He did not have a history of DISH or ankylosing spondylitis. Unlike the usual presentation, in 2016 the patient first noticed dysphagia with liquids, specifically the feeling of residue in the throat after swallowing. Over a period of two years he began to experience the same symptoms with food, and eventually, with pills. He reported no difference in severity of symptoms based on positioning or time of day.

During this time, the severity of the dysphagia was alleviated by diet modification including changing the solid consistency of food to a softer consistency, such as slow-cooked meat. However, the patient did not change the way he was drinking liquids, and so continued to experience dysphagia-like symptoms including a globus sensation and "frequent" coughing. He continued to take his medications with thin liquids. He reported occasions of being out walking and beginning to cough, at times expectorating pieces of food. Based on the patient's report, he was on the equivalent of a minced and moist diet (International Dysphagia Diet Standardization Initiative IDDSI 5) with thin liquids (IDDSI 0).

The patient stated he first reported his progressive dysphagia symptoms to his primary care physician in August 2019. He then underwent an Esophagogastroduodenoscopy (EGD) in December 2019 but the procedure was unsuccessful as the esophagus could not be intubated. He did not receive any speech/dysphagia therapy during this time other than a MBSS on March 3 and 18th, 2020 at another facility. Subsequently between March and August 2020, the patient reports having three Fiberoptic Endoscopic Evaluation of Swallow (FEES) procedures. Results from these procedures are unavailable but patient reports they "didn't fix anything".

The patient on cervical CT was diagnosed with large C3-C4-C5 osteophytes, which is known to cause inflammatory hyperplasia and irritate the pharynx [23]. He underwent C3-C4-C5 osteophytectomy by neurosurgery on 8/12/20 with ENT assistance due to difficult intubation. Postprocedurally, he was extubated but needed to be re-intubated due to airway edema and respiratory failure, similar to a case presented by Lin et al. [24]. A tracheostomy was placed on 8/13/20 postop day 1 due to inability to extubate. He was started on Decadron due to airway edema. He was given a cervical collar postoperatively which was to be worn at all times. On 8/15/20 post-op day 3 the patient was unable to speak with a Passy Muir Valve (PMV) due to weakness and drop in oxygen saturation. He failed a swallow evaluation. On post-op day 5 patient had his tracheostomy cuff deflated and was able to communicate with PMV in place. A repeat swallow evaluation at bedside with water was completed which showed immediate blue dye return with strong cough reflex revealing overt aspiration with sensory awareness. The swallow evaluation was discontinued due to high aspiration risk and the patient was made strict NPO. Due to persistent dysphagia, a gastrostomy tube was placed on post-op day 6 (8/18/20). Repeat EGD occurred on 8/18/20, after the osteophytectomy, as patient failed extubation as well as the swallow study. At this time the scope was unable to be passed due to oropharyngeal narrowing from extrinsic compression. On $8 / 19 / 20$ the tracheostomy was changed to cuffless. On post-op day $8(8 / 20 / 20)$, he was admitted to acute rehabilitation and at that time he was able to tolerate a Passy-Muir Valve (PMV) daily. The patient had a persistent mild cough following surgery. 


\section{Ethics Statement}

This case report complies with the Research Ethics Guidelines. Consent was obtained on 9/14/20 by the individual being reviewed, in an effort to help others suffering from similar symptoms and considering surgical intervention.

\section{Clinical Course in Acute Rehabilitation}

\subsection{Materials/Methods}

Consent for participation in this study was obtained from the patient prior to his discharge from our acute inpatient rehabilitation unit. An extensive chart review confirmed that the patient had a "long history" of dysphagia secondary to cervical osteophytes. Per review of notes from a previous facility, the patient underwent an MBSS on 3/3/20 which was listed as a "failure." Following surgery, the record further stated the patient "failed swallow eval" on 8/16/20, failed blue dye test on $8 / 17 / 20$, and that SLP recommended resuming dysphagia therapy after decannulation.

At the time of his clinical swallow evaluation the patient presented with tracheostomy (Shiley, type 7) and PMV in place. Patient was on $5 \mathrm{~L}$ oxygen via a trach mask and had a hoarse, weak voice and weak cough indicating a compromised airway. He required frequent oral suctioning, indicating an inability to manage secretions due to dysphagia. The patient could provide his medical history, but did have some mild confusion and cognitive deficits when first encountered, scoring 24/30 on the St Louis University Mental Status Exam (SLUMS) which indicates mild neurocognitive impairment given the patient's educational level [25].

On the oral-mechanism exam the patient's dentition was within normal limits, but presented with decreased labial and lingual range of motion due to deconditioning, hyponasal resonance thought to be related to his prior uvulopalatopharyngoplasty and impaired respiratory support for speech and cough. Speech intelligibility was $60 \%$ at the sentence level and both volitional and reflexive coughs were weak.

Clinical swallow evaluation revealed moderate oral dysphagia and severe pharyngeal dysphagia marked by impaired laryngeal elevation upon palpation, poor secretion management, need for oral and deep suctioning, and consistent wet vocal quality. Recommendations were to continue NPO status with alternative means of nutrition via PEG tube. The patient was placed on aspiration precautions and PO trials with SLP only. Blue dye testing or repeat MBSS were deemed unsafe due to the severity of dysphagia and deferred at the time of the evaluation.

Dysphagia therapy was indicated given the patient's clinical presentation and was therefore provided by the SLP one hour per day, five days per week, for thirty total sessions. Therapy focused on secretion management, breathing techniques including incentive spirometer, oral hygiene, blue dye testing, pharyngeal strengthening exercises including Masako maneuver, modified Shaker, tongue protrusion and range of motion exercises, base of tongue strengthening, thermal stimulation, and later PO trials of ice chips. Secondary goals wer improved vocal hygiene due to persistent hoarseness and hyponasality utilizing vocal exercises and oral-motor exercises including isometrics.

The patient was motivated and was diligent with the exercises, but remained NPO throughout his rehab stay. The patient failed blue dye testing on multiple occasions throughout his stay. The patient's respiratory function improved from $5 \mathrm{~L}$ oxygen via trach mask $(8 / 21 / 20)$ to $2 \mathrm{~L}$ oxygen via 
trach mask (8/28/20) to room air with $2 \mathrm{~L}$ oxygen at night time $(9 / 2 / 20)$ to the trach being red capped $(9 / 8 / 20)$. The patient became less reliant on oral suctioning as he was better able to swallow or expectorate his secretions. The patient was discharged home with a Shiley 7 cuffless trach on 9/14/20 following a MBSS with recommendation for continued NPO and ice chip/water trials following the Frazier Water Protocol (FWP) [26].

In individuals with ACOs, it is difficult to assess if pharyngeal residue is a result of muscular weakness or edema from surgery. As mentioned previously, an MBSS was completed on 9/11/20 prior to the patient's discharge home. At the time of the evaluation, the patient was NPO receiving all of his nourishment through a PEG tube. The patient demonstrated mild oral dysphagia with soft solids, such as minced and moist consistencies, evidenced by reduced bolus control and maintenance in the oral cavity due to lingual weakness. Through the course of his dysphagia rehabilitation the patient improved his endurance, respiratory and lingual strength for better bolus management particularly for liquids. The pharyngeal stage of swallow was severely affected with primary concern for lack of epiglottic inversion, which resulted in reduced airway protection and placed the patient at significant aspiration risk. The epiglottis is a structure in the neck that inverts during swallow to assist with airway protection during swallow, protecting liquid or solid material from entering into the trachea. According to Vose \& Humbert [27], pharyngeal constriction, tongue base contraction, and epiglottic elevation together facilitate epiglottic inversion so that laryngeal vestibule closure is possible. The same authors further suggest that there is normal variability in laryngeal vestibule closure.

\section{Results/Outcomes}

Based on interpretation of the MBSS completed on 9/11/2020, this patient was noted to have pharyngeal edema, which resulted in the epiglottis making contact with the posterior pharyngeal wall during the swallow, impeding the passage of the bolus through the pharynx. This reduced pharyngeal pressure and residue remained in the valleculae and piriform sinus cavities of the pharynx. The patient reflexively attempted to clear the residue with multiple dry swallows but was unsuccessful, resulting in eventual penetration into the glottis. Penetration is indicative of the bolus entering into the laryngeal vestibule above the trachea. Aspiration occurred with both thin liquids (Figure 1) and moderately thick liquids (Figure 2), which are also referred to as honey thick liquids. Aspiration was silent, indicating an insensate larynx potentially resulting from intubation and/or surgery. Reduced opening of the upper esophageal sphincter occurred due to impaired pharyngeal pressures and laryngeal elevation affecting clearance of the bolus from the pharynx into the esophagus. This resulted in backflow at the pharyngoesophageal segment. Pharyngeal pressure was further compromised by the patient's reduced velopharyngeal closure and resulted in residue on the pharyngeal wall at the point of lingual contact. 


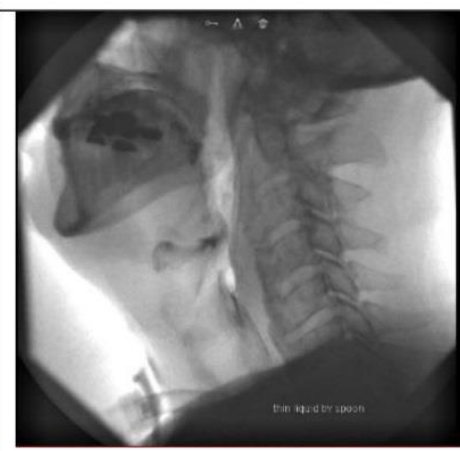

Before Swallow

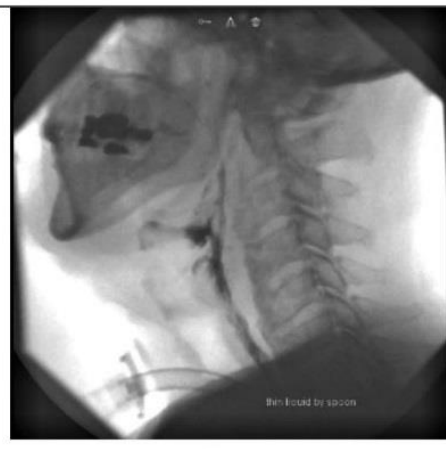

During Swallow

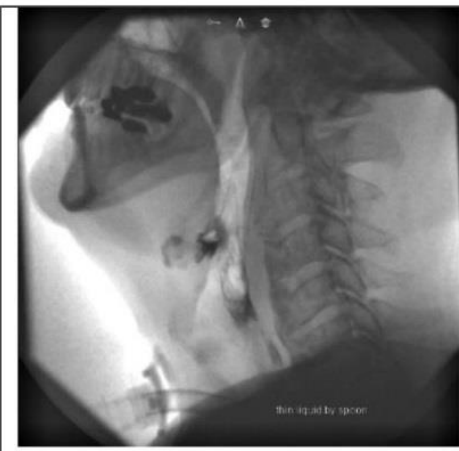

After Swallow

Figure 1 Thin Liquids via Teaspoon, MBSS 9/11/2020.

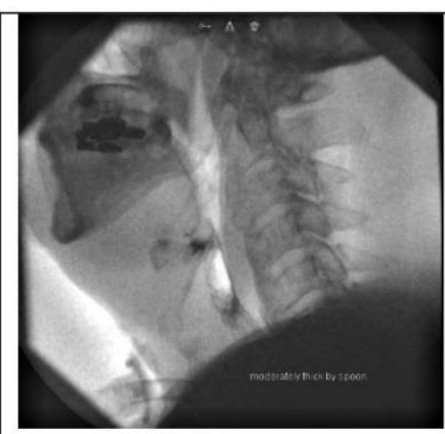

Before Swallow

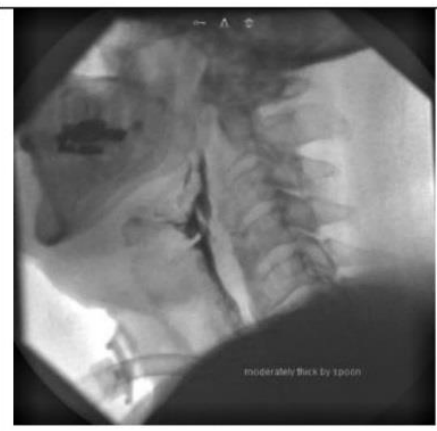

During Swallow

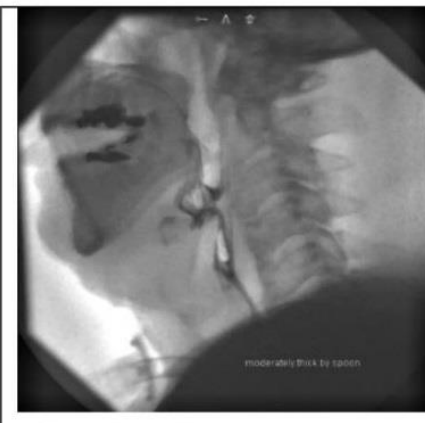

After Swallow

Figure 2 Moderately Thick Liquids via Teaspoon, MBSS 9/11/2020.

The patient tolerated $2 \mathrm{cc}$ thin liquid by spoon without penetration or aspiration. When the bolus amount was increased to $5 \mathrm{cc}$, the patient had penetration to the vocal folds (VF) that was not ejected and was therefore aspirated after the swallow when the vocal folds were abducted. This was followed by a reflexive cough. Penetration to the vocal folds occurred with all consistencies with trace silent aspiration, aspiration without a reflexive cough response, occurring with moderately thick liquids. Thin liquids were more easily cleared than thicker consistencies, which is frequently noted with patients suffering from osteophytes or other spinal deformities. There is much evidence documented about the impact of bolus volume and viscosity on swallow and muscle activation [28-32]. The patient needed to swallow six or more times to clear $80 \%$ of the pharyngeal residue. Swallow maneuvers, such as the effortful swallow, were not helpful in clearing residue. The patient demonstrated the ability for PO intake of thin liquids by spoon with controlled amounts. These results indicated that the patient remained at significant risk for aspiration one-month postsurgery, with aspiration noted across two consistencies. Per protocol at our facility, the patient was placed on the FWP [26].

Based on the Bolus Residue Scale (BRS), which was first presented by Rommel et al. [33], the patient received a score of 4 (Residue in valleculae and posterior pharyngeal wall or piriform sinus) for thin, slightly thick, mildly thick, puree and minced and moist consistencies, but received a score 
of 6 (Residue in valleculae and posterior pharyngeal wall and piriform sinus) for moderately thick liquids (Figure 2).

The Penetration Aspiration Scale (PenAsp) was utilized as a measurement of the MBSS [34]. It indicates the adequacy of airway protection during swallowing on a scale from 1 (material does not enter the airway) to 8 (material enters the airway, passes below the vocal folds, and no effort is made to eject), judging the depth of bolus penetration or aspiration into the larynx or below the vocal folds and the presence or effectiveness of the airway protective response. The patients' results on the PenAsp are shown in Table 1 for the different food consistencies. Unfortunately records from MBSS performed prior to admission and post discharge are not available for comparison and the only data received is that the patient "failed" with a recommendation for NPO.

Table 1 PenAsp scores for different food consistencies.

\begin{tabular}{ll}
\hline PenAsp Score & Consistency \\
\hline 5 & Minced and Moist \\
5 & Puree \\
$1,5,7$ & Thin liquids \\
5 & Slightly Thick liquids \\
1,5 & Mildly Thick liquids \\
5,8 & Moderately Thick liquids \\
\hline
\end{tabular}

The Dysphagia Outcome Severity Score (DOSS) is based on a scale looking at the level of independence, level of nutrition, as well as diet level and diet modifications. Level of independence is divided into seven levels according to the Functional Independence Measure (FIM) model and linked to severity. The initial levels are 7- within normal limits, 6- modified independence, 5- distant supervision, 4- intermittent supervision, 3- total supervision, 2-maximum assistance, and 1 dependent/NPO nutrition. The level of nutrition scale was divided into two possible recommendations for nutrition and were linked to severity level: levels 7-3 (full oral nutrition) and levels 2-1 (non-oral nutrition). Finally, diet level and diet modifications guidelines identify oral intake as follows: levels 7-6 (normal diet consistency), level 5 (may need one diet consistency restriction), level 4 (one to two diet consistency restrictions), and level 3 (two or more diet consistency restrictions) [35].

The patient received a DOSS score of 2 based on his FIM score of 1, the severity of his dysphagia, aspiration occurring across 2 consistencies, severe pharyngeal residue that he was unable to fully clear, and the need to continue with tube feeding (TF). He remained NPO with the goal to safely initiate some PO intake beginning with ice chips and sips of water and slowly advancing to other thin liquids as he continued with his therapy in outpatient. 
The FOIS is a scale that identifies a patient's oral or non-oral intake according to the textures that he or she can tolerate [36]. This patient's FOIS improved from a score of 1 to 2 .

Throughout treatment sessions the patient demonstrated good adherence to treatment, following written exercises independently that were at visible points in his room. Initially the patient was confused and stated his thinking felt "foggy" but this was also targeted and improved during the course of his rehabilitation. Although the patient was initially impulsive, there was no evidence later that he was non-compliant with NPO or other precautions.

\section{Conclusions, Discussions, and Limitations}

In this unique case study, a clinical course was presented for a patient with chronic dysphagia as a result of ACOs status post an osteophytectomy. This patient is unlike the typical patient with DISH or ankylosing spondylitis as he had large C3-C4-C5 osteophytes as a result of degenerative aging. His case was further complicated by a history of uvulopalatopharyngoplasty and carotid endarterectomy resulting in a more complex and atypical anatomy. Additionally, his initial clinical presentation of dysphagia with difficulty swallowing liquids versus solids was atypical for most individuals. Although anti-inflammatories were initially given, they were discontinued post-op 1 week and therefore may have contributed to persistent airway compromise and pharyngeal edema, resulting in the need for prolonged tracheostomy. As stated above, surgical intervention for symptomatic ACOs is rare, thus the current case study adds to the current literature pool consisting of isolated case studies while considering the complexities of this particular case. We observed that while the patient was motivated and had a good response to therapy, he remained NPO throughout his rehab stay.

There is limited consistent evidence for determination of optimal surgical candidates who return to PO intake as their primary source of nutrition. Additionally, post-surgical complications including impact of anesthesia and compromised respiratory status on recovery are minimally discussed. There is large variability in the literature for recovery time frames, return to PO/diet consistencies, and outcome measures such as quality of life making it difficult for patients to determine their optimal course of action.

Decision making for surgical intervention is multifactorial and should consider comorbid medical issues that may affect recovery, the depth of the osteophyte, prognosis for recovery, potential for recurrence, the plan for post procedure recovery, discharge planning related to PEG and trach management, as well as physical assistance needed due to deconditioning related to the procedure. Further consideration should be given to possible cognitive issues impacting patients' ability to participate in rehabilitation and follow diet recommendations and maneuvers.

There is limited evidence for pre/post dysphagia interventions and currently no specific guidelines for dysphagia treatment for individuals with ACO [2]. Given that an optimal treatment method has not been agreed upon, it is difficult for SLPs to come to a consensus regarding the appropriate plan of care for patients affected by ACOs. The current case study adds to the body of literature that suggests that empirical research is needed to measure dysphagia treatment outcomes.

It is also important to consider the important role of the SLP in proactive assessment and therapy when working with individuals with ACOs. SLPs are integral in evaluating swallow function by way 
of videofluoroscopy and prescribing appropriate compensatory strategies and physiological exercises to assist with improved swallow function.

As was noted in the current case study, previous hospital reports provided little to no information about MBSS results and dysphagia therapy conducted. Lack of interdisciplinary communication across settings results in poor understanding of a patient's medical history. It is unknown what recommendations were made prior to the patient undergoing surgery. There is a possibility that all strategies and exercises were not exhausted prior to the surgery. In addition, some patients may not undergo an MBSS prior to surgery, which may lead to inappropriate or missed opportunities of diet modifications or therapeutic intervention that may eliminate the need for surgical intervention. It is the role of the SLP to advocate for instrumental evaluations for patients with ACOs prior to surgical intervention.

Limitations of this study include biases inherent to a retrospective case study and also the presence of only one participant. Lack of videofluoroscopy follow-up also serves as a limitation as it prevents the current research team from investigating long-term improvements of swallow function. This case study poses the question as to whether surgical intervention is an optimal solution for all patients. As was found in Chung et al. [3], 18\% of patients reported recurrence of symptoms approximately one-year post-surgery. Miyamoto et al. [37] recommend follow up for 10 years postsurgery due to osteophyte regrowth in all of their patients of $1 \mathrm{~mm}$ per year. Outcomes of surgery as they come from existing literature need to be provided to patients prior to participating in surgical intervention. This educational responsibility undoubtedly falls upon the physician and SLP.

\section{Acknowledgments}

The authors would like to thank Dr. Loverso and the Casa Colina Board of Directors, the Inpatient Speech Department, and the Casa Colina Hospital and Centers for Rehabilitation Foundation for supporting this research.

\section{Author Contributions}

$\mathrm{MH}, \mathrm{JP}$ and SH provided the patient case report, diagnostic and treatment protocols. Along with these 3 authors, SG conducted the research and drafted the paper. ER provided feedback and edits of the drafted paper as well as developed tables for representation of data.

\section{Funding}

This case report is supported by Casa Colina Hospital and Centers for Rehabilitation.

\section{Competing Interests}

The authors have declared that no competing interests exist.

\section{References}

1. Albayrak I, Bağcacı S, Sallı A, Kucuksen S, Uğurlu H. A rare cause of dysphagia: Compression of the esophagus by an anterior cervical osteophyte due to ankylosing spondylitis. Korean J Intern Med. 2013; 28: 614-618. 
2. Choi HE, Jo GY, Kim WJ, Do HK, Kwon JK, Park SH. Characteristics and clinical course of dysphagia caused by anterior cervical osteophyte. Ann Rehabil Med. 2019; 43: 27-37.

3. Chung YS, Zhang HY, Ha Y, Park JY. Surgical outcomes of dysphagia provoked by diffuse idiopathic skeletal hyperostosis in the cervical spine. Yonsei Med J. 2020; 61: 341-348.

4. Kim Y, Park GY, Seo YJ, Im S. Effect of anterior cervical osteophyte in poststroke dysphagia: A case-control study. Arch Phys Med Rehabil. 2015; 96: 1269-1276.

5. Lecerf $\mathrm{P}, \mathrm{Malard} \mathrm{O}$. How to diagnose and treat symptomatic anterior cervical osteophytes? Eur Ann Otorhinolaryngol Head Neck Dis. 2010; 127: 111-116.

6. McGarrah PD, Teller D. Posttraumatic cervical osteophytosis causing progressive dysphagia. Nan Fang Yi Ke Da Xue Xue Bao. 1997; 90: 858-860.

7. Egerter AC, Kim ES, Lee DJ, Liu JJ, Cadena G, Panchal RR, et al. Dysphagia secondary to anterior osteophytes of the cervical spine. Global Spine J. 2015; 5: 78-83.

8. Ozgursoy OB, Salassa JR, Reimer R, Wharen RE, Deen HG. Anterior cervical osteophyte dysphagia: Manofluorographic and functional outcomes after surgery. Head Neck. 2010; 32: 588-593.

9. Kolz JM, Alvi MA, Bhatti AR, Tomov MN, Bydon M, Sebastian AS, et al. Anterior cervical osteophyte resection for treatment of dysphagia. Global Spine J. 2020. Doi: $10.1177 / 2192568220912706$.

10. Laus M, Malaguti MC, Alfonso C, Ferrari D, Zappoli FA, Giunti A. Dysphagia due to cervical osteophytosis. La Chirurgia degli organi di movimento. 1995; 80: 263-271.

11. American Speech-Language-Hearing Association. Knowledge and skills needed by speechlanguage pathologists performing videofluoroscopic swallowing studies [Internet]. Rockville, MA: American Speech-Language-Hearing Association; 2004. Available from: https://www.asha.org/policy/KS2004-00076/.

12. Kumar M, Shahi PB, Adsul N, Acharya S, Kalra KL, Chahal RS. Progressive dysphagia and dysphonia secondary to DISH-related anterior cervical osteophytes: A case report. Surg Neurol Int. 2020; 11: 69.

13. Maiuri F, Stella L, Sardo L, Buonamassa S. Dysphagia and dyspnea due to an anterior cervical osteophyte. Arch Orthop Trauma Surg. 2002; 122: 245-247.

14. Mallepally AR, Tandon V, Chhabra HS. Dysphagia in a young adult: Rare case of giant cervical osteophyte. Asian J Neurosurg. 2020; 15: 218-221.

15. Nicolas H, Voelker A, Jarvers JS, Gulow J, Heyde CE. Results after the surgical treatment of anterior cervical hyperostosis causing dysphagia. Eur Spine J. 2015; 24: 489-493.

16. Węgłowski R, Piech P. Dysphagia as a symptom of anterior cervical hyperostosis-case report. Ann Agric Environ Med. 2019; 27: 314-316.

17. Jeong $\mathrm{H}$, Seo HG, Han TR, Chung CK, Oh BM. Kinematic changes in swallowing after surgical removal of anterior cervical osteophyte causing dysphagia: A case series. Ann Rehabil Med. 2014; 38: 865-870.

18. Carlson ML, Archibald DJ, Graner DE, Kasperbauer JL. Surgical management of dysphagia and airway obstruction in patients with prominent ventral cervical osteophytes. Dysphagia. 2011; 26: 34-40.

19. Hamouda WO. Timing for surgical intervention in DISHphagia. J Craniovertebr Junction Spine. 2018; 9: 227-231. 
20. Kapetanakis S, Vasileiadis I, Papanas N, Goulimari R, Maltezos E. Can a giant cervical osteophyte cause dysphagia and airway obstruction? A case report. Wien Klin Wochenschr. 2011; 123: 291293.

21. Oppenlander ME, Orringer DA, La Marca F, McGillicuddy JE, Sullivan SE, Chandler WF, et al. Dysphagia due to anterior cervical hyperosteophytosis. Surg Neurol. 2009; 72: 266-270.

22. Shin HW, Jang JC, Lim HH, Park MK, Bae GE, Choi SU, et al. Postoperative respiratory difficulty due to asymptomatic anterior cervical osteophyte after brain tumor surgery: A case report. Korean J Anesthesiol. 2016; 69: 640-643.

23. Matan AJ, Hsu J, Fredrickson BA. Management of respiratory compromise caused by cervical osteophytes: A case report and review of the literature. Spine J. 2002; 2: 456-459.

24. Lin HW, Quesnel AM, Holman AS, Curry Jr WT, Rho MB. Hypertrophic anterior cervical osteophytes causing dysphagia and airway obstruction. Ann Otol Rhinol Laryngol. 2009; 118: 703-707.

25. Morley JE, Tumosa N. Saint Louis University mental status examination (SLUMS). Aging Success. 2002; 12: 4.

26. Panther K. The Frazier free water protocol. Dysphagia. 2005; 14: 4-9.

27. Vose A, Humbert I. "Hidden in plain sight": A descriptive review of laryngeal vestibule closure. Dysphagia. 2019; 34: 281-289.

28. Dantas RO, Kern MK, Massey BT, Dodds WJ, Kahrilas PJ, Brasseur JG, et al. Effect of swallowed bolus variables on oral and pharyngeal phases of swallowing. Am J Physiol Gastrointest Liver Physiol. 1990; 258: G675-G681.

29. Hiss SG, Strauss M, Treole K, Stuart A, Boutilier S. Effects of age, gender, bolus volume, bolus viscosity, and gustation on swallowing apnea onset relative to lingual bolus propulsion onset in normal adults. J Speech Lang Hear Res. 2004; 47: 572-583.

30. Nascimento WV, Cassiani RA, Santos CM, Dantas RO. Effect of bolus volume and consistency on swallowing events duration in healthy subjects. J Neurogastroenterol Motil. 2015; 21: 78.

31. Regueiro MR, Nascimento WV, Parreira LC, Dantas RO. Videofluoroscopic analysis of different volumes of liquid bolus swallowing in healthy individuals: Comparison between height and sex. Clinics. 2017; 72: 693-697.

32. Wu S, Chu L, Liu CF, Zhang Q, Zhang YF, Zhou TF, et al. Effect of changes in bolus viscosity on swallowing muscles in patients with dysphagia after stroke. Chin Med J. 2018; 131: 2868.

33. Rommel N, Borgers C, Van Beckevoort D, Goeleven A, Dejaeger E, Omari TI. Bolus residue scale: An easy-to-use and reliable videofluoroscopic analysis tool to score bolus residue in patients with dysphagia. Int J Otolaryngol. 2015; 2015: 780197.

34. Rosenbek JC, Robbins JA, Roecker EB, Coyle JL, Wood JL. A penetration-aspiration scale. Dysphagia. 1996; 11: 93-98.

35. O'Neil KH, Purdy M, Falk J, Gallo L. The dysphagia outcome and severity scale. Dysphagia. 1999; 14: 139-145.

36. Crary MA, Mann GD, Groher ME. Initial psychometric assessment of a functional oral intake scale for dysphagia in stroke patients. Arch Phys Med Rehabil. 2005; 86: 1516-1520.

37. Miyamoto K, Sugiyama S, Hosoe H, linuma N, Suzuki Y, Shimizu K. Postsurgical recurrence of osteophytes causing dysphagia in patients with diffuse idiopathic skeletal hyperostosis. Eur Spine J. 2009; 18: 1652-1658. 


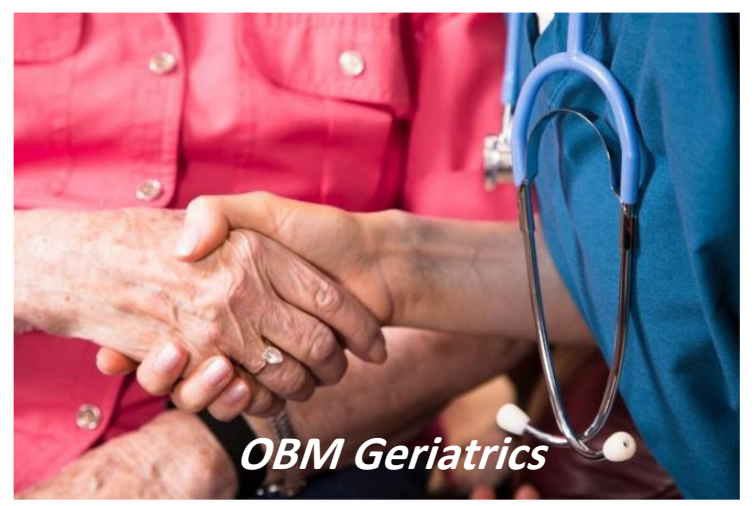

Enjoy OBM Geriatrics by:

1. Submitting a manuscript

2. Joining in volunteer reviewer bank

3. Joining Editorial Board

4. Guest editing a special issue

For more details, please visit:

http://www.lidsen.com/journals/geriatrics 